\title{
Biomarkers in Heart Failure
}

\author{
Yasuchika TAKEISHI, ${ }^{1} \mathrm{MD}$
}

\begin{abstract}
SUMMARY
Appropriate use of biomarkers is clinically important for identifying heart failure in its early stage, optimizing risk stratification, and managing patients. This article describes established and traditional biomarkers as well as novel biomarkers reflective of myocardial stress, myocardial damage, extracellular matrix, oxidative stress, inflammation, renal function, micro RNAs, and heart failure with preserved left ventricular ejection fraction. This review focuses on the recent advances in cardiac and non-cardiac biomarkers of heart failure and their appropriate use in clinical practice. (Int Heart J 2014; 55: 474-481)
\end{abstract}

Key words: BNP, NT-proBNP, ST2, Troponin, H-FABP, Galectin-3, Cystatin C, Pentraxin 3

$\mathrm{C}$ ardiovascular diseases are the predominant cause of death in developed countries. Among the afflicted, patients with heart failure are increasing, and this complex syndrome is becoming a public health problem, especially with the aging of populations. To establish diagnostic, therapeutic, and prognostic strategies, the identification of reliable biomarkers for heart failure is necessary. ${ }^{1,2)}$ Although a number of biomarkers have been developed, the ideal biomarkers should meet certain criteria: 1) non-invasive sample collection, 2) a high degree of sensitivity and specificity, 3) able to detect the disease at an early stage, 4) sensitivity high enough to reflect relevant changes in disease conditions, 5) a long halflife within the sample, 6) rapid measurement system responding to clinical needs, and 7) low cost. ${ }^{3,4)}$ In this article, the focus will be on the recent advances in cardiac and non-cardiac biomarkers of heart failure and their appropriate use in clinical practice.

\section{Myocardial Stress Markers}

B-type natriuretic peptide: B-type natriuretic peptide (BNP) is a well-established biomarker, extensively used for the diagnosis and prognosis of patients with heart failure. BNP is a cardiac hormone, identified as the second compound of the natriuretic peptide family and secreted predominantly from the ventricle in response to mechanical overload. ${ }^{5)}$ In the failing heart, increased wall stress and neurohormonal activation facilitate BNP secretion chiefly from ventricular myocytes. BNP promotes diuresis, vasodilatation, and attenuation of renin and aldosterone secretion. Tsutamoto, et al initially measured plasma levels of BNP in 85 patients with chronic heart failure and a left ventricular ejection fraction (LVEF) of less than $0.45{ }^{6}{ }^{6}$ The plasma levels of BNP increased in proportion to the severity of heart failure. Plasma levels of BNP were 5-fold higher in non-survivors than in survivors. Among clinical and hemody- namic parameters, Cox proportional hazard analysis revealed that only plasma BNP $(P<0.0001)$ and pulmonary capillary wedge pressure $(P=0.003)$ were significant independent predictors of mortality in patients with heart failure. Plasma levels of BNP provided prognostic information independent of other variables previously associated with a poor prognosis. To date, a number of studies have reported the diagnostic and prognostic impacts of BNP in heart failure. ${ }^{7,8)}$

Beyond the prognostic value of a single BNP measurement, monitoring changes in BNP concentrations over time may be helpful for further risk stratification. Many physicians measure plasma BNP for the diagnosis, risk stratification, and monitoring of heart failure. In the Val-HeFT trial, changes in BNP over time were associated with corresponding changes in mortality and morbidity in 4,305 patients. ${ }^{9)}$ The change from baseline to 4 and 12 months in BNP was analyzed by quartiles for subsequent mortality and the first morbid event. Baseline BNP in quartiles showed a quartile-dependent increase in mortality and the first morbid event. Importantly, patients with the greatest percentage decrease in BNP from baseline to 4 and 12 months had the lowest mortality and first morbid event whereas patients with the greatest percentage increase in BNP had the highest mortality and first morbid event. Understanding these potentials for modulation with treatment may fuel interest in serial measurements of BNP so as to guide and improve heart failure therapy. ${ }^{10)}$

Although BNP-guided monitoring of patients with heart failure has been reported, ${ }^{11)}$ it is still controversial as to whether serial measurements of natriuretic peptide are useful in heart failure management. ${ }^{12,13)}$ Jourdain, et al evaluated the prognostic impact of a therapeutic strategy using plasma BNP levels. ${ }^{14)}$ A total of 220 New York Heart Association (NYHA) functional class II and III patients considered optimally treated with angiotensin-converting enzyme inhibitors, $\beta$-blockers, and diuretics were randomly assigned either to medical treatment ac-

From the ${ }^{1}$ Department of Cardiology and Hematology, Fukushima Medical University, Fukushima, Japan.

Address for correspondence: Yasuchika Takeishi, MD, Department of Cardiology and Hematology, Fukushima Medical University, 1 Hikarigaoka, Fukushima 9601295, Japan. E-mail: takeishi@fmu.ac.jp

Received for publication August 14, 2014. Accepted August 23, 2014

Released in advance online on J-STAGE October 24, 2014.

All rights reserved by the International Heart Journal Association. 
cording to current guidelines (clinical group), or to a group with the goal of decreasing plasma BNP levels to less than 100 $\mathrm{pg} / \mathrm{mL}$ (BNP group). Both groups had similar baseline clinical characteristics. At the end of the first 3 months, all types of drugs were changed more frequently in the BNP group. Mean dosages of angiotensin-converting enzyme inhibitors and $\beta$-blockers were significantly higher in the BNP group $(P<$ 0.05). Importantly, during follow-up (median 15 months), fewer patients in the BNP group reached the combined endpoint, heart failure-related death or hospital stay, than in the clinical group (24\% versus 52\%, $P<0.001$ ). Similarly, in the PROTECT study, N-terminal pro-B-type natriuretic peptide (NTproBNP)-guided patients had more frequent drug changes, and greater dose increases of therapies with improved mortality. ${ }^{15)}$ On the contrary, several trials reported no difference in medical management and prognosis between the 2 arms. ${ }^{16,17)}$ In addition, if the BNP-guided approach is effective even in study populations for whom the target level of BNP is not achieved, it is unclear whether BNP adds incremental value to the determination of therapy. ${ }^{13)}$ It should be noted that in the Systolic Heart Failure Treatment Supported by BNP (STARS-BNP) trial, patients in the BNP-guided arm had twice the number of physician visits and more frequent medication changes than those in the standard care arm, even though only $33 \%$ reached the target BNP of less than $100 \mathrm{pg} / \mathrm{mL}^{14)}$

N-terminal pro-B-type natriuretic peptide: The main stimulus for proBNP synthesis and secretion from cardiomyocytes is mechanical stretch. ${ }^{18,19)}$ On secretion, the propeptide is split into the biologically active BNP and the remaining part is the prohormone NT-proBNP. In the failing heart, ventricular NTproBNP synthesis is markedly increased. Compared to BNP, NT-proBNP has a longer half-life and is biologically inert, but both are secreted into the plasma in equimolar quantities. ${ }^{18,19)}$

A diagnostic and prognostic value of NT-proBNP for systolic heart failure was examined in the general population (382 women and 290 men). ${ }^{20)}$ NT-proBNP identified patients with symptoms of heart failure and LVEF $\leq 40 \%$ with a sensitivity of 0.92 , a specificity of 0.86 , positive and negative predictive values of 0.11 and 1.00, and an area under the curve (AUC) of 0.94. NT-proBNP was the strongest independent predictor of mortality (hazard ratio, 5.70; $P<0.0001$ ) and hospital admissions for heart failure (hazard ratio, 13.83; $P<$ $0.0001)$.

The utility of NT-proBNP for predicting the occurrence of death and hospitalization was prospectively evaluated in patients with severe heart failure. ${ }^{21)}$ Baseline plasma concentrations of NT-proBNP were measured in a subgroup of 814 men and 197 women with symptoms, at rest or on minimal exertion, who were enrolled in the COPERNICUS study. By univariate Cox regression analysis, NT-proBNP was found to be a powerful predictor of all-cause mortality (relative risk, 2.7; 95\% confidence interval $(\mathrm{CI}), 1.7-4.3 ; P=0.0001$ for above versus below median) and all-cause mortality or hospitalization for heart failure (relative risk, $2.4 ; 95 \% \mathrm{CI}, 1.8-3.4 ; P=$ 0.0001 for above versus below median).

ST2: Using genomic technology, ST2, an interleukin-1 receptor family member, was identified as a gene that is markedly induced by mechanical stretch in cardiomyocytes. ${ }^{22)}$ The soluble receptor form of ST2 is secreted and detectable in human circulation.

Weinberg, et al initially demonstrated that serum soluble
ST2 is a novel biomarker for myocardial stress and neurohormonal activation in patients with heart failure. ${ }^{23)}$ Serum levels of ST2 were measured with enzyme-linked immunosorbent assay (ELISA) on samples from 161 patients obtained at trial enrollment and 2 weeks after the enrollment of the PRAISE-2 trial. ST2 levels were correlated with levels of BNP $(r=0.36$, $P<0.0001)$ and norepinephrine $(r=0.39, P<0.0001)$. The change in ST2 was a univariate predictor of subsequent mortality or transplantation $(P=0.048)$. In multivariate models including BNP, the change in ST2 remained significant as a predictor of mortality or transplantation independent of BNP. Januzzi, et al measured concentrations of ST2 in 593 dyspneic patients with and without acute heart failure in an emergency department. ${ }^{24)}$ Concentrations of ST2 were higher among those with acute heart failure compared with those without $(0.50$ versus $0.15 \mathrm{ng} / \mathrm{mL}, P<0.001)$. Median concentrations of ST2 at presentation to the emergency department were higher among decedents than survivors at 1 year $(1.08$ versus $0.18 \mathrm{ng}$ / $\mathrm{mL}, P<0.001)$. In multivariable analyses, an ST2 concentration $\geq 0.20 \mathrm{ng} / \mathrm{mL}$ strongly predicted death at 1 year in dyspneic patients (hazard ratio, 5.6; 95\% CI, 2.2 - 14.2; $P<0.001$ ).

\section{Myocardial Damage Markers}

Troponins: The progression of heart failure is associated with a progressive loss of cardiomyocytes that can be detected clinically as continuously increased serum levels of troponins. Although troponin $\mathrm{T}$ is a sensitive and specific marker of acute coronary syndrome, Setsuta, et $a l^{25)}$ and Sato, et $a l^{26)}$ reported that troponin $\mathrm{T}$ was also detected in severe heart failure patients and associated with adverse clinical outcomes. In the Acute Decompensated Heart Failure National Registry (ADHERE), troponins were measured at the time of admission in 67,924 patients who were hospitalized for acute decompensated heart failure. ${ }^{27)}$ Overall, 4,240 patients $(6.2 \%)$ were positive for either troponin T or I. As shown in Figure 1, patients who were positive for troponins had higher in-hospital mortality than those who were negative for troponin $(8.0 \%$ versus $2.7 \%$, $P<0.001)$. The adjusted odds ratio for death in the group of patients with a positive troponin test was 2.55 (95\% CI, 2.24 2.89; $P<0.001)$.

Sensitive assays of troponins have expanded the clinical applications of troponin testing. ${ }^{28)}$ Latina, et al have reported that troponin $\mathrm{T}$ is detected in more than $90 \%$ of outpatients with stable chronic heart failure. ${ }^{29)}$ With a highly sensitive assay, a dose-dependent association of troponins and risk for cardiac death has been found in these study populations at levels below the detection threshold of the standard assay. The Dallas Heart Study, a population-based study with a highly sensitive assay, demonstrated that troponin $\mathrm{T}$ is detectable in $25 \%$ of the general population, and that those with positive troponin $\mathrm{T}$ are associated with left ventricular hypertrophy, systolic dysfunction, high all-cause, and cardiovascular mortality. ${ }^{30)}$

On the other hand, cardiac troponin $\mathrm{T}$ is detectable in the circulation of patients with non-cardiac diseases such as infection and stroke, using highly sensitive assays. ${ }^{31,32)}$ Although the detailed mechanisms are still unknown, Nakamura, et al have recently reported that patients with positive cardiac troponin $\mathrm{T}$ are associated with high non-cardiac mortality as well as cardiovascular mortality in patients with heart failure. ${ }^{33)}$

Heart type-fatty acid binding protein (H-FABP): H-FABP, a 


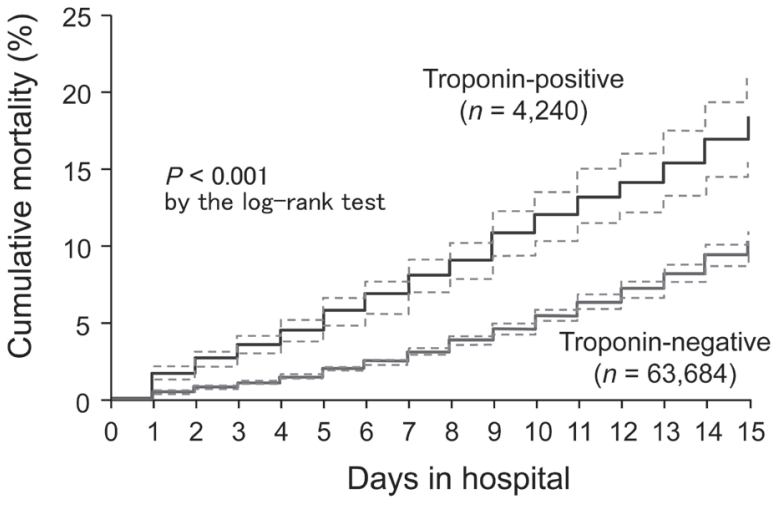

Figure 1. Mortality according to number of days in the hospital and troponin status at presentation. $P<0.001$ by the log-rank test. Dashed lines show 95\% confidence intervals. (Modified; Peacock WF 4th, et al. N Engl J Med 2008; 358: 2117-26. ${ }^{27)}$ )

low molecular weight (14-15 kDa) cytoplasmic and nonenzymatic protein which transports long-chain fatty acids into the cardiomyocyte, is rapidly released into the circulation from the damaged myocardium. ${ }^{34)}$ Therefore, H-FABP has been used as an early and sensitive diagnostic marker for acute myocardial infarction. On the other hand, it has been demonstrated that the serum level of H-FABP is increased in patients with advanced heart failure, and H-FABP is a marker for myocardial cell injury and prognosis in chronic heart failure. ${ }^{35)}$

Niizeki, et al measured serum H-FABP and troponin T levels in 126 consecutive heart failure patients at hospital admission. ${ }^{36}$ They found that the positive rate of H-FABP was higher than that of troponin $\mathrm{T}$ in all heart failure patients $(46 \%$ [58/126] versus 26\% [33/126], $P<0.0001)$, and in severe heart failure (NYHA III/IV) patients (69\% [34/49] versus $47 \%$ [23/49], $P=0.0121)$. The area under the receiver operating characteristic (ROC) curve was higher for H-FABP than for troponin T ( 0.779 versus $0.581, P=0.009)$, suggesting that $\mathrm{H}$ FABP had greater predictive capacity to identify high-risk patients than troponin T. However, it should be noted that serum H-FABP levels were affected by age, gender, obesity, and renal function in a large group of volunteers $(n=2,099) .{ }^{37)}$ These effects should be taken into account in determining appropriate reference values for H-FABP.

\section{Extracellular Matrix}

Matrix metalloproteinase: Plasma levels of matrix metalloproteinase (MMP), a key determinant of extracellular matrix degradation, are increased in heart failure. The Framingham Heart Study showed that plasma MMP-9 levels were associated with increased left ventricular end-diastolic dimension and increased wall thickness, indicating that plasma MMP-9 level may be a marker for cardiac extracellular matrix degradation and left ventricular remodeling. ${ }^{38)}$

Radauceanu, et al measured serum levels of amino-terminal propeptide of collagen III, MMP-1, the tissue inhibitor of metalloproteinase-1 (TIMP-1), and interleukins in 1,009 patients enrolled in the Research into Etanercept Cytokine Antagonism in Ventricular Dysfunction (RECOVER) trial. ${ }^{39)} \mathrm{A}$ positive correlation was detected between the 2 classes of markers (amino-terminal propeptide of collagen III to inter-

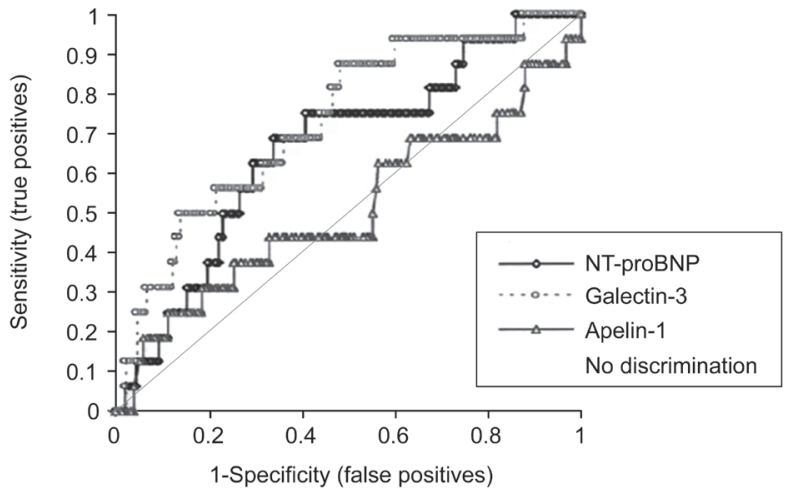

Figure 2. Combined receiver operating characteristic curves for aminoterminal pro-brain natriuretic peptide (NT-proBNP), galectin-3 and apelin for 60-day mortality in heart failure. (van Kimmenade RR, et al. J Am Coll Cardiol 2006; 48: 1217-24. ${ }^{41)}$ )

leukin-18, TIMP-1 to interleukin-18, and MMP-1 to interleukin-10). In the adjusted multivariable model including all biomarkers, only the amino-terminal propeptide of collagen III $(P=0.03)$ and MMP-1 $(P=0.048)$ were independent predictors of the 6-minute walk test. Amino-terminal propeptide of collagen III $(P=0.001)$ was the only biomarker independently associated with death and hospitalization due to heart failure. These data suggest that excessive extracellular matrix turnover is associated with cardiac remodeling, functional capacity deterioration, and poor outcome in heart failure. ${ }^{39)}$

Galectin-3: Galectin-3, a member of a large family of $\beta$-galactoside-binding animal lectins and derived from macrophages, interacts with various ligands located at the extracellular matrix, including laminin, collagen, and integrins. ${ }^{40)}$ Expression of galectin-3 is markedly increased in hypertrophied and failing hearts, and galectin-3 induces cardiac fibroblast proliferation, collagen deposition, and ventricular dysfunction.

Van Kimmenade, et al measured plasma levels of NTproBNP and galectin-3 in 599 patients presenting with dyspnea at the emergency department. ${ }^{41)}$ Levels of galectin-3 were higher in subjects with heart failure than in those without. The ROC analysis to predict 60-day mortality showed that galectin-3 had a greater AUC $(0.74, P=0.0001)$ than NT-proBNP $(0.67, P=0.009)$ as shown in Figure 2. In a multivariate logistic regression analysis, an elevated level of galectin-3 was the best independent predictor of 60-day mortality (odds ratio $10.3 ; P<0.01)$ or the combination of death/recurrent heart failure within 60 days (odds ratio 14.3; $P<0.001$ ).

\section{Oxidative Stress}

8-hydroxy-2'-deoxyguanosine: Oxidative stress is known to play a crucial role in the pathogenesis of heart failure. DNA in the nucleus is one of the major targets of reactive oxygen species, and 8-hydroxy-2'-deoxyguanosine (8-OHdG) is produced from deoxyguanosine in DNA by reactive oxygen species and used as a marker of oxidative DNA damage. ${ }^{42)}$ Kobayashi, et al showed that urinary $8-\mathrm{OHdG}$ was higher in heart failure patients than in control subjects, and that urinary $8-\mathrm{OHdG}$ became higher as the NYHA class increased. ${ }^{43)}$ Moreover, there was a significant correlation between urinary $8-\mathrm{OHdG}$ and cardiac functional parameters. 
Suzuki, et al measured serum 8-OHdG levels in 230 patients with chronic heart failure and 42 control subjects without heart failure by a sandwich ELISA. ${ }^{44)}$ Serum $8-O H d G$ concentrations were higher in patients with heart failure than in control subjects $(P<0.001)$, and increased with advancing NYHA functional class $(P<0.001)$. Kaplan-Meier survival curves demonstrated that the cardiac event rate was markedly higher in patients with high $8-\mathrm{OHdG}$ levels than in those with normal 8 -OHdG levels $(62.4 \%$ versus $29.6 \%, P=0.0007)$. Serum 8 -OHdG levels provide important prognostic information for the risk stratification of patients with heart failure.

Neopterin: Neopterin is produced by activated monocytes/ macrophages upon stimulation with interferon- $\gamma$. The amounts of neopterin correlate with the capacity of activated monocytes/macrophages to release reactive oxygen species. ${ }^{45)}$ Thus, neopterin concentrations in body fluids can be regarded as an indirect estimate of the degree of oxidative stress emerging during cell-mediated immune response.

Sasaki, et al measured the serum neopterin concentration in 198 patients with heart failure and 62 control subjects by a competitive ELISA. ${ }^{46)}$ Serum concentration of neopterin increased with advancing NYHA functional class $(P<0.001)$. The 4th quartile of neopterin concentration was associated with the highest risk of cardiac events (11.1-fold) compared to the 1st quartile. In the multivariate Cox analysis, serum neopterin concentration was an independent risk factor for cardiac events (hazard ratio 1.70; 95\% CI $1.16-2.50 ; P=0.0068$ ).

\section{Inflammation}

C-reactive protein: Activation of the inflammatory system plays an important role in the pathogenesis of heart failure. Increased plasma levels of tumor necrosis factor- $\alpha$ and interleukin- 6 in patients with heart failure are related to decreasing functional status and provide important prognostic information for morbidity and mortality. ${ }^{47,48)}$ One of the inflammatory markers, C-reactive protein (CRP), is produced in the liver in response to stimulation of various cytokines, mostly interleukin-6.

It has been reported that elevated high-sensitive CRP has an independent prognostic value in heart failure patients. Yin, et al measured serum levels of high-sensitive CRP in 108 patients with chronic heart failure and LVEF of less than $50 \%{ }^{49)}$ In a multivariate analysis, LVEF and serum levels of high-sensitive CRP were independent significant predictors for adverse outcomes in these patients (hazard ratio, 3.714; $P=0.024$, and hazard ratio, 2.584; $P=0.047$, respectively). Mueller, et al evaluated the prognostic role of inflammation among 214 consecutive patients presenting with acute heart failure at the emergency department. ${ }^{50)}$ Patients in the highest CRP tertile significantly more often required admission to the intensive care unit $(33 \%$ versus $14 \%, P=0.028)$ and died in hospital (15\% versus $2 \%, P=0.027)$ compared to the first tertile. After multivariate adjustment, CRP remained an independent predictor of death (hazard ratio, 1.4; 95\% CI, $1.1-1.8 ; P=0.044$ ). Kamioka, et al examined whether high-sensitive CRP level before cardiac re-synchronization therapy (CRT) implantation was able to predict the response to CRT and cardiac deaths in severe heart failure patients. ${ }^{51)}$ High-sensitive CRP level was significantly higher in non-responders than in responders to CRT $(P<0.01)$. Multivariate logistic regression analysis showed an independent relationship between high-sensitive CRP and the incidence of non-responders to CRT (odds ratio: $1.499, P=0.011$ ). Stepwise multivariate Cox proportional hazard analysis identified the high-sensitive CRP level as the strongest predictive factor for cardiac death (hazard ratio: $1.337, P=0.001)$.

Pentraxin 3: Pentraxin 3 is a member of the long pentraxin family, and conserves the C-terminal pentraxin domain with the classical short pentraxins, but differs by an unrelated long $\mathrm{N}$-terminal domain. ${ }^{52,53)}$ A variety of cell types including dendritic cells, mononuclear phagocytes, macrophages, smooth muscle cells, fibroblasts, and endothelial cells can produce pentraxin 3 upon exposure to primary inflammatory signals such as interleukin- 1 , tumor necrosis factor- $\alpha$, oxidized lowdensity lipoprotein, microbial moieties, and agonists for different members of the Toll-like receptor family. ${ }^{52,53)}$

Suzuki, et al measured the plasma pentraxin 3 levels in 196 patients with heart failure and 60 control subjects without heart failure by a sandwich ELISA. ${ }^{54)}$ The cardiac event-free rate was markedly lower in patients with high pentraxin 3 levels than in those with normal pentraxin 3 levels $(44.7 \%$ versus $89.2 \%, P<0.0001)$. As shown in the Table, multivariate Cox proportional hazard analysis demonstrated that the plasma pentraxin 3 level (per one standard deviation increase), but not tumor necrosis factor- $\alpha$, was an independent predictor of cardiac events (hazard ratio, 1.20; 95\% CI, $1.03-1.40 ; P=0.0162$ ). Patients were divided into 4 groups based on plasma pentraxin 3 values from the 1 st to 4 th quartile. The highest 4 th quartile of plasma pentraxin 3 levels was associated with the highest risk

Table. Results of the Multivariate Cox Proportional Hazard Analysis for Cardiac Death and Re-Hospitalization

\begin{tabular}{lccc}
\hline Variable & Hazard ratio & 95\% Confidence interval & $P$ \\
\hline Age, per 5-year increase & 1.16 & $0.98-1.38$ & 0.0904 \\
LVEDD, per 9.8 mm increase & 1.47 & $1.06-2.05$ & 0.0207 \\
LVEF, per 18.1\% increase & 0.99 & $0.69-1.40$ & 0.9467 \\
BNP, per 762 pg/mL increase & 1.25 & $1.00-2.14$ & 0.0493 \\
Estimated GFR, per 32.2 mL increase & 1.12 & $0.70-1.83$ & 0.6424 \\
Uric acid, per 2.0 mg/dL increase & 1.20 & $0.90-1.59$ & 0.1989 \\
hs-CRP, per 1.35 mg/dL increase & 1.24 & $1.01-1.53$ & 0.0418 \\
TNF- $\alpha$, per 1.02 pg/mL increase & 1.16 & $0.88-1.53$ & 0.2971 \\
Pentraxin 3, per 6.22 ng/mL increase & 1.20 & $1.03-1.40$ & 0.0162 \\
\hline
\end{tabular}

LVEDD indicates left ventricular end-diastolic diameter; LVEF, left ventricular ejection fraction; BNP, B-type natriuretic peptide; GFR, glomerular filtration rate; hs-CRP, high-sensitive C-reactive protein; and TNF, tumor necrosis factor. (Suzuki $\mathrm{S}$, et al. Am Heart J 2008; 155: 75-81. ${ }^{54)}$ ) 
of cardiac events (9.23-fold compared with the 1st quartile).

\section{Renal Function}

Creatinine and estimated glomerular filtration rate: A number of studies have reported a strong association between renal dysfunction and mortality in heart failure, and the concept of cardio-renal syndrome has been widely recognized. ${ }^{55)}$ Studies in patients with stable heart failure have shown that the serum creatinine level predicts mortality from progressive heart failure independently of established prognostic variables. Estimated glomerular filtration rate (GFR) from the Cockcroft-Gault or Modification of Diet in Renal Disease (MDRD) equation is a strong predictor of mortality in heart failure. ${ }^{56)}$ Hillege, et al reported that in 372 patients with chronic heart failure, baseline GFR was the most powerful predictor of mortality among other risk factors including NYHA functional class, the use of angiotensin-converting enzyme inhibitors, and LVEF. ${ }^{57)}$ Patients in the lowest quartile of GFR values ( $<44 \mathrm{~mL} / \mathrm{minute}$ ) had almost 3 times the risk of mortality (hazard ratio, $2.85 ; P<$ $0.001)$ of patients in the highest quartile ( $>76 \mathrm{~mL} /$ minute).

Cystatin C: Cystatin C, a 13-kD basic protein, is a cysteine protease inhibitor involved in the catabolism of proteins. Cystatin $\mathrm{C}$ is produced in all nucleated cells at a constant rate and is freely filtered by the glomerulus without secretion or subsequent reabsorption to the blood flow. ${ }^{58)}$ Therefore, cystatin $C$ is a serum measure of renal function that appears to be independent of age, sex, and lean muscle mass.

Shlipack, et al measured serum cystatin $\mathrm{C}$ levels in samples collected from 4,637 participants in the Cardiovascular Health Study, a cohort study of elderly persons living in the community. ${ }^{59)}$ As the levels of cystatin $\mathrm{C}$ increased, hazard ratio for death increased after multivariate adjustment (Figure 3). They also showed in elderly persons with heart failure that cystatin $\mathrm{C}$ is a stronger predictor of mortality than creatinine. ${ }^{60)}$ In the study by Arimoto, et al, Cox multivariate proportional hazard analysis revealed that the cystatin $\mathrm{C}$ level was the independent predictor for cardiac events (hazard ratio, 2.93; $95 \%$ CI, $1.29-6.64 ; P<0.01$ ), and that the cardiac event rate was markedly higher in patients with an elevated cystatin $\mathrm{C}$ level than in those with a normal level $(44.2 \%$ versus $13.4 \%, P<$ $0.001){ }^{61)}$ Furthermore, in patients with normal creatinine levels $(n=91)$, the cardiac event rate was similarly higher in patients with elevated cystatin $\mathrm{C}$ than in those with normal levels (36.4\% versus $10.0 \%, P=0.003)$.

\section{Combination of Several Biomarkers}

Myocardial stress, damage, and fibrosis: Combination of biomarkers that reflect different pathogenic aspects in heart failure would provide incremental diagnostic and prognostic value. Niizeki, et al studied whether the combination of markers for myocardial stress and damage at admission can reliably risk stratify patients hospitalized for chronic heart failure. ${ }^{62)}$ Circulating levels of H-FABP and BNP were measured at admission in 186 consecutive patients hospitalized for chronic heart failure. A stepwise Cox regression analysis demonstrated that high H-FABP (hazard ratio 5.416; $P=0.0002$ ) and high BNP (hazard ratio $2.411 ; P=0.0463$ ) were independent predictors of cardiac events. High levels of both H-FABP and $\mathrm{BNP}$ at admission were associated with the highest incidence

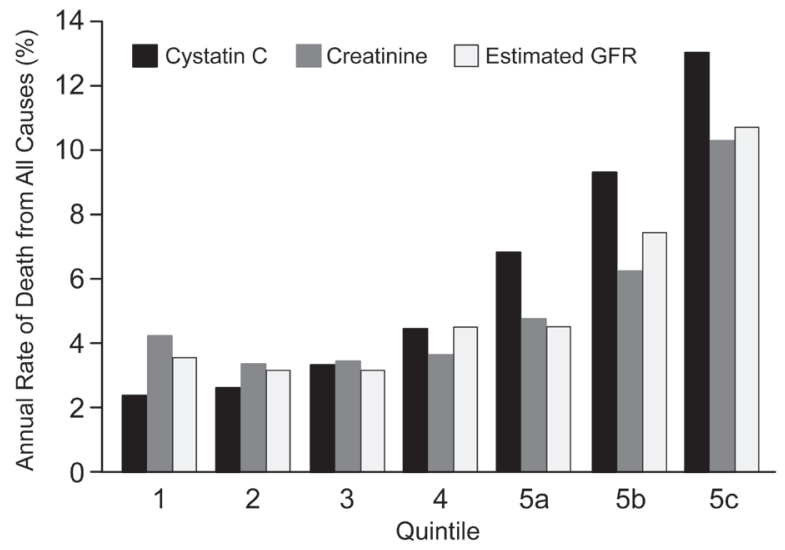

Figure 3. All cause mortality according to quintile of renal function by cystatin C, creatinine, and estimated glomerular filtration rate (GFR). The fifth quintile was subdivided into three roughly equal groups, labeled $5 \mathrm{a}$, 5b, and 5c. (Shlipak MG, et al. N Engl J Med 2005; 352: 2049-60. ${ }^{59)}$ )

in cardiac mortality and cardiac events. Kaplan-Meier analysis also showed that a combination of H-FABP and BNP levels could reliably stratify patients for cardiac events.

In the HF-ACTION (Heart Failure: A Controlled Trial Investigating Outcomes of Exercise Training) trial, Ahmed, et al studied whether biomarkers of myocardial stress and fibrosis improved the prediction of death in patients with chronic heart failure. NT-proBNP and galectin-3 levels were assessed at baseline in 813 patients with chronic heart failure due to left ventricular systolic dysfunction $(\mathrm{LVEF} \leq 35 \%){ }^{63)}$ Elevations of two biomarkers were associated with increased risk for both pump failure and sudden cardiac death. Clinical variables along with NT-proBNP levels were stronger predictors of pump failure (C statistic: 0.87$)$ than sudden cardiac death $(\mathrm{C}$ statistic: 0.73 ). Addition of galectin-3 led to an improved net risk classification of $11 \%$ for sudden cardiac death, but not pump failure. Clinical predictors along with NT-proBNP levels were strong predictors of pump failure risk, with insignificant incremental contributions of galectin- 3 . $^{63)}$

Model for End-Stage Liver Disease (MELD) score: Liver dysfunction due to heart failure is often referred to as cardiac or congestive hepatopathy. Recently, the composite Model for End-Stage Liver Disease (MELD) scoring series, with types such as MELD (including total bilirubin, creatinine, prothrombin time-international ratio (INR)) and MELD-XI (MELD excluding INR) have been developed. ${ }^{64,65)}$ They are established scoring systems for liver or hepato-renal function, and a high score is associated with a poor prognosis, not only in patients having undergone liver transplantation, but also in those with advanced heart failure possibly requiring heart transplantation and/or a ventricular assist device.

In HF patients, MELD scoring indicates multiple organ dysfunction secondary to impaired cardiac function. Abe, et al recently analyzed 562 patients who were admitted for the treatment of decompensated heart failure. ${ }^{66)}$ A MELD-XI score was graded, and patients were divided into two groups based on the median MELD-XI score. They showed that rates of cardiac death, non-cardiac death, and all-cause death were significantly higher in the high MELD-XI group than in the low MELD-XI group. In Cox proportional hazard analysis, a high MELD-XI 
score was found to be an independent predictor of cardiac death and all-cause mortality in heart failure patients. The MELD-XI scoring system, simply calculated from total bilirubin and creatinine, can identify high-risk patients having multiple organ failure associated with heart failure. ${ }^{66)}$

\section{Circulating MicroRNAs}

It has been recognized that microRNAs (miRNAs) are differentially expressed in the failing heart and control a variety of cellular processes essential to the heart. ${ }^{3,67)}$ On the other hand, miRNAs are reportedly present in the circulation and are found to be stable in plasma. ${ }^{68)}$ Since their identification in the circulation, miRNAs have attracted much interest as novel biomarkers of cardiovascular diseases. ${ }^{3,69)}$ To date, several reports have shown that circulating miRNAs demonstrate different profiles in heart failure compared to controls, and could be used as biomarkers for this syndrome.

Tijsen, et al showed that miR423-5p was specifically enriched in the blood in patients with heart failure and was related to the diagnosis and severity of heart failure. ${ }^{70)}$ ROC curve analysis showed that miR423-5p distinguished patients with heart failure from control subjects with an area under the curve of $0.91(P<0.001)$. Goren, et al revealed from meticulous screening of 186 miRNAs that circulating levels of 4 prominent miRNAs (miR423-5p, miR320a, miR22, and miR92b) were increased in heart failure patients. ${ }^{71)}$ There was a significant association between the miRNA-score, which was defined using the levels of these 4 miRNAs, and several important known prognostic parameters, including BNP levels, a wide QRS duration, and dilatation of the left ventricle and left atrium. However, Tutarel, et al failed to find elevations of miR423$5 \mathrm{p}$ levels in patients after atrial repair for transposition of the great arteries. ${ }^{72)}$ Differences in study population and right ventricular overload in the study by Tutarel, et al, might be causes for this discrepancy.

Some other miRNAs were found to be linked to the diagnosis of heart failure. Corsten, et al reported that circulating levels of miR499 and miR122 were increased in acute heart failure. ${ }^{73)}$ Ellis, et al. investigated the diagnostic utility of miRNAs in differentiating between patients with heart failure and non-heart failure-related breathlessness. ${ }^{74)}$ Four miRNAs, miR103, miR142-3p, miR30b, and miR342-3p, were differentially expressed between HF and controls, chronic obstructive pulmonary disease, and other breathless patients.

Taken together, circulating miRNAs are novel candidate biomarkers for heart failure and large-scale prospective clinical trials comparing them with known biomarkers are necessary to validate their true clinical utility.

\section{Biomarkers for Heart Failure With Preserved Left Ventricular Ejection Fraction}

Heart failure with preserved left ventricular ejection fraction $(\mathrm{HFpEF})$ comprises a growing proportion of overall heart failure. HFpEF relates to the complex interactions between ventricular diastolic dysfunction, ventricular-vascular stiffening, and comorbid illnesses such as hypertension, atrial fibrillation, diabetes, and chronic kidney disease. ${ }^{75)}$ Efficient diagnostic and prognostic biomarkers remain undetermined in this population.
Biomarkers most extensively examined in HFpEF include myocardial stress and extracellular matrix biomarkers. ${ }^{76)}$ Yamaguchi, et al initially reported that an elevation of BNP was a diagnostic marker of diastolic heart failure among subjects with preserved systolic function, independent of the degree of left ventricular hypertrophy. ${ }^{77)}$ Myocardial fibrosis evidenced by increased extracellular matrix collagen is a key pathological feature of $\mathrm{HFpEF}$, and circulating collagen markers may reflect this excess fibrosis. ${ }^{75)}$ Kitahara, et al measured carboxy-terminal telopeptide of type I collagen, a marker of collagen degradation, at admission in 156 consecutive patients hospitalized for chronic heart failure. ${ }^{78)}$ They found that carboxy-terminal telopeptide of type I collagen was an independent predictor of cardiac deaths and hospitalizations in HFpEF (hazard ratio, 1.210; 95\% CI, 1.013-1.446; $P<0.05$ ). Krum, et al measured plasma levels of procollagen type I amino-terminal peptide and procollagen type III amino-terminal peptide in 334 patients with HFpEF in the substudy of the Irbesartan in Heart Failure With Preserved Systolic Function (I-PRESERVE) trial. ${ }^{79)}$ For each $10 \mu \mathrm{g} / \mathrm{L}$ increase in procollagen type I amino-terminal peptide, the hazard ratio for the primary endpoint was 1.09 (95\% CI, $1.052-1.13 ; P<0.0001)$. For each $10 \mu \mathrm{g} / \mathrm{L}$ increase in procollagen type III amino-terminal peptide, the hazard ratio was $2.47(95 \% \mathrm{CI}, 0.97-6.33 ; P=$ 0.059). In addition, several biomarkers, including inflammation, growth differentiation factor 15 , cystatin C, NT-proBNP, and galectin-3, were associated with development and clinical outcomes of HFpEF. ${ }^{75)}$

Appropriate use of biomarkers is clinically important for identifying heart failure in its early stage, optimizing risk stratification, and managing patients. ${ }^{80)}$ This article has described established and traditional biomarkers such as BNP, NT-pro BNP and troponins as well as novel biomarkers reflective of myocardial stress, myocardial damage, extracellular matrix, oxidative stress, inflammation, cardio-renal pathophysiology, miRNAs, and HFpEF. Since numerous novel biomarkers have been reported in the literature, a systematic approach aimed directly at identifying the clinical benefit for patients with heart failure is needed.

\section{REFERENCES}

1. Braunwald E. Biomarkers in heart failure. N Engl J Med 2008; 358: 2148-59. (Review)

2. Maisel AS, Choudhary R. Biomarkers in acute heart failure--state of the art. Nat Rev Cardiol 2012; 9: 478-90. (Review)

3. Tijsen AJ, Pinto YM, Creemers EE. Circulating microRNAs as diagnostic biomarkers for cardiovascular diseases. Am J Physiol Heart Circ Physiol 2012; 303: H1085-95. (Review)

4. Choudhary R, Iqbal N, Khusro F, Higginbotham E, Green E, Maisel A. Heart failure biomarkers. J Cardiovasc Transl Res 2013; 6: 471-84. (Review)

5. Mukoyama M, Nakao K, Hosoda K, et al. Brain natriuretic peptide as a novel cardiac hormone in humans: evidence for an exquisite dual natriuretic peptide system, atrial natriuretic peptide and brain natriuretic peptide. J Clin Invest 1991; 87: 1402-12.

6. Tsutamoto T, Wada A, Maeda K, et al. Attenuation of compensation of endogenous cardiac natriuretic peptide system in chronic heart failure: prognostic role of plasma brain natriuretic peptide concentration in patients with chronic symptomatic left ventricular dysfunction. Circulation 1997; 96: 509-16.

7. Yu CM, Sanderson JE. Plasma brain natriuretic peptide--an inde- 
pendent predictor of cardiovascular mortality in acute heart failure. Eur J Heart Fail 1999; 1: 59-65.

8. Koglin J, Pehlivanli S, Schwaiblmair M, Vogeser M, Cremer P vonScheidt W. Role of brain natriuretic peptide in risk stratification of patients with congestive heart failure. J Am Coll Cardiol 2001; 38: 1934-41.

9. Anand IS, Fisher LD, Chiang YT, et al. Changes in brain natriuretic peptide and norepinephrine over time and mortality and morbidity in the Valsartan Heart Failure Trial (Val-HeFT). Circulation 2003; 107: 1278-83.

10. Felker GM, Hasselblad V, Hernandez AF, O'Connor CM. Biomarker-guided therapy in chronic heart failure: a meta-analysis of randomized controlled trials. Am Heart J 2009; 158: 422-30.

11. Troughton RW, Frampton CM, Yandle TG, Espiner EA, Nicholls MG, Richards AM. Treatment of heart failure guided by plasma aminoterminal brain natriuretic peptide (N-BNP) concentrations. Lancet 2000; 355: 1126-30.

12. Januzzi JL, Troughton R. Are serial BNP measurements useful in heart failure management? Serial natriuretic peptide measurements are useful in heart failure management. Circulation 2013; 127: 500-7. (Review)

13. Desai AS. Are serial BNP measurements useful in heart failure management? Serial natriuretic peptide measurements are not useful in heart failure management: the art of medicine remains long. Circulation 2013; 127: 509-16.

14. Jourdain P, Jondeau G, Funck F, et al. Plasma brain natriuretic peptide-guided therapy to improve outcome in heart failure: the STARS-BNP Multicenter Study. J Am Coll Cardiol 2007; 49: 1733-9.

15. Januzzi JL Jr, Rehman SU, Mohammed AA, et al. Use of aminoterminal pro-B-type natriuretic peptide to guide outpatient therapy of patients with chronic left ventricular systolic dysfunction. J Am Coll Cardiol 2011; 58: 1881-9.

16. Eurlings LW, van Pol PE, Kok WE, et al. Management of chronic heart failure guided by individual N-terminal pro-B-type natriuretic peptide targets: results of the PRIMA (Can PRo-brain-natriuretic peptide guided therapy of chronic heart failure IMprove heart fAilure morbidity and mortality?) study. J Am Coll Cardiol 2010; 56: 2090-100.

17. Karlström P, Alehagen U, Boman K, Dahlström U; UPSTEPStudy Group. Brain natriuretic peptide-guided treatment does not improve morbidity and mortality in extensively treated patients with chronic heart failure: responders to treatment have a significantly better outcome. Eur J Heart Fail 2011; 13: 1096-103.

18. Hunt PJ, Yandle TG, Nicholls MG, Richards AM, Espiner EA. The amino-terminal portion of pro-brain natriuretic peptide (ProBNP) circulates in human plasma. Biochem Biophys Res Commun 1995; 214: 1175-83.

19. Pemberton CJ, Yandle TG, Rademaker MT, Charles CJ, Aitken GD, Espiner EA. Amino-terminal proBNP in ovine plasma: evidence for enhanced secretion in response to cardiac overload. Am J Physiol 1998; 275: H1200-8.

20. Groenning BA, Raymond I, Hildebrandt PR, Nilsson JC, Baumann M, Pedersen F. Diagnostic and prognostic evaluation of left ventricular systolic heart failure by plasma $\mathrm{N}$-terminal pro-brain natriuretic peptide concentrations in a large sample of the general population. Heart 2004; 90: 297-303.

21. Hartmann F, Packer M, Coats AJ, et al. Prognostic impact of plasma N-terminal pro-brain natriuretic peptide in severe chronic congestive heart failure: a substudy of the Carvedilol Prospective Randomized Cumulative Survival (COPERNICUS) trial. Circulation 2004; 110: 1780-6.

22. Weinberg EO, Shimpo M, De Keulenaer GW, et al. Expression and regulation of ST2, an interleukin-1 receptor family member, in cardiomyocytes and myocardial infarction. Circulation 2002; 106: 2961-6.

23. Weinberg EO, Shimpo M, Hurwitz S, Tominaga S, Rouleau JL, Lee RT. Identification of serum soluble ST2 receptor as a novel heart failure biomarker. Circulation 2003; 107: 721-6.

24. Januzzi JL Jr, Peacock WF, Maisel AS, et al. Measurement of the interleukin family member ST2 in patients with acute dyspnea: results from the PRIDE (Pro-Brain Natriuretic Peptide Investigation of Dyspnea in the Emergency Department) study. J Am Coll Cardiol 2007; 50: 607-13.

25. Setsuta K, Seino Y, Takahashi N, et al. Clinical significance of elevated levels of cardiac troponin $\mathrm{T}$ in patients with chronic heart failure. Am J Cardiol 1999; 84: 608-11.

26. Sato Y, Yamada T, Taniguchi R, et al. Persistently increased serum concentrations of cardiac troponin $\mathrm{t}$ in patients with idiopathic dilated cardiomyopathy are predictive of adverse outcomes. Circulation 2001; 103: 369-74.

27. Peacock WF 4th, De Marco T, Fonarow GC, et al. Cardiac troponin and outcome in acute heart failure. N Engl J Med 2008; 358: 2117-26.

28. Lemos JA. Increasing sensitive assays for cardiac troponins: a review. JAMA 2013; 309: 2262-9. (Review)

29. Latini R, Masson S, Anand IS, et al. Prognostic value of very low plasma concentrations of troponin $\mathrm{T}$ in patients with stable chronic heart failure. Circulation 2007; 116: 1242-9.

30. de Lemos JA, Drazner MH, Omland T, et al. Association of troponin $\mathrm{T}$ detected with a highly sensitive assay and cardiac structure and mortality risk in the general population. JAMA 2010; 304: 2503-12.

31. Roongsritong C, Warraich I, Bradley C. Common causes of troponin elevations in the absence of acute myocardial infarction: Incidence and clinical significance. Chest 2004; 125: 1877-84. (Review)

32. Agewall S, Giannitsis E, Jernberg T, Katus H. Troponin elevation in coronary vs. non-coronary disease. Eur Heart J 2011; 32: 40411. (Review)

33. Nakamura Y, Yoshihisa A, Takiguchi M, et al. High-sensitivity cardiac troponin $\mathrm{T}$ predicts non-cardiac mortality in heart failure. Cir J 2014; 78: 890-5.

34. Glatz JF, Klein AH, van Nieuwenhoven FA, Hermens WT, van Dieijen-Visser MP, van Vusse GJ. Fatty-acid-binding protein as a plasma marker for the estimation of myocardial infarct size in humans. Br Heart J 1994; 71: 135-40.

35. Arimoto T, Takeishi Y, Shiga R, et al. Prognostic value of elevated circulating heart-type fatty acid binding protein in patients with congestive heart failure. J Card Fail 2005; 11: 56-60.

36. Niizeki T, Takeishi Y, Arimoto T, et al. Heart-type fatty acid-binding protein is more sensitive than troponin $\mathrm{T}$ to detect the ongoing myocardial damage in chronic heart failure patients. J Card Fail 2007; 13: 120-7.

37. Niizeki T, Takeishi Y, Takabatake N, et al. Circulating levels of heart-type fatty acid-binding protein in a general Japanese population: effects of age, gender, and physiologic characteristics. Circ J 2007; 71: 1452-7.

38. Sundström J, Evans JC, Benjamin EJ, et al. Relations of plasma matrix metalloproteinase-9 to clinical cardiovascular risk factors and echocardiographic left ventricular measures: the Framingham Heart Study. Circulation 2004; 109: 2850-6.

39. Radauceanu A, Ducki C, Virion JM, et al. Extracellular matrix turnover and inflammatory markers independently predict functional status and outcome in chronic heart failure. J Card Fail 2008; 14: 467-74.

40. Sharma UC, Pokharel S, van Brakel TJ, et al. Galectin-3 marks activated macrophages in failure-prone hypertrophied hearts and contributes to cardiac dysfunction. Circulation 2004; 110: 3121-8.

41. van Kimmenade RR, Januzzi JL Jr, Ellinor PT, et al. Utility of amino-terminal pro-brain natriuretic peptide, galectin-3, and apelin for the evaluation of patients with acute heart failure. J Am Coll Cardiol 2006; 48: 1217-24.

42. Lagorio S, Tagesson C, Forastiere F, Iavarone I, Axelson O, Carere A. Exposure to benzene and urinary concentrations of 8-hydroxydeoxyguanosine, a biological marker of oxidative damage to DNA. Occup Environ Med 1994; 51: 739-43.

43. Kobayashi S, Susa T, Tanaka T, et al. Urinary 8-hydroxy-2'-deoxyguanosine reflects symptomatic status and severity of systolic dysfunction in patients with chronic heart failure. Eur J Heart Fail 
2011; 13:29-36.

44. Suzuki S, Shishido T, Ishino M, et al. 8-Hydroxy-2'-deoxyguanosine is a prognostic mediator for cardiac event. Eur J Clin Invest 2011; 41: 759-66.

45. Müller MM, Curtius HC, Herold M, Huber CH. Neopterin in clinical practice. Clin Chim Acta 1991; 201: 1-16. (Review)

46. Sasaki T, Takeishi Y, Suzuki S, et al. High serum level of neopterin is a risk factor of patients with heart failure. Int J Cardiol 2010; 145: 318 .

47. Rauchhaus M, Doehner W, Francis DP, et al. Plasma cytokine parameters and mortality in patients with chronic heart failure. Circulation 2000; 102: 3060-7.

48. Kinugawa T, Kato M, Yamamoto K, Hisatome I, Nohara R. Proinflammatory cytokine activation is linked to apoptotic mediator, soluble Fas level in patients with chronic heart failure. Int Heart J 2012; 53: 182-6.

49. Yin WH, Chen JW, Jen HL, et al. Independent prognostic value of elevated high-sensitivity $\mathrm{C}$-reactive protein in chronic heart failure. Am Heart J 2004; 147: 931-8.

50. Mueller C, Laule-Kilian K, Christ A, Brunner-La Rocca HP, Perruchoud AP. Inflammation and long-term mortality in acute congestive heart failure. Am Heart J 2006; 151: 845-50.

51. Kamioka M, Suzuki H, Yamada S, Kamiyama Y, Saitoh S, Takeishi Y. High sensitivity C-reactive protein predicts nonresponders and cardiac deaths in severe heart failure patients after CRT implantation. Int Heart J 2012; 53: 306-12.

52. Alles VV, Bottazzi B, Peri G, Golay J, Introna M, Mantovani A. Inducible expression of PTX3, a new member of the pentraxin family, in human mononuclear phagocytes. Blood 1994; 84: 348393.

53. Garlanda C, Bottazzi B, Bastone A, Mantovani A. Pentraxins at the crossroads between innate immunity, inflammation, matrix deposition, and female fertility. Annu Rev Immunol 2005; 23 : 337-66. (Review)

54. Suzuki S, Takeishi Y, Niizeki T, et al. Pentraxin 3, a new marker for vascular inflammation, predicts adverse clinical outcomes in patients with heart failure. Am Heart J 2008; 155: 75-81.

55. Silverberg DS, Wexler D, Blum M, Iaina A. The cardio renal anemia syndrome: correcting anemia in patients with resistant congestive heart failure can improve both cardiac and renal function and reduce hospitalizations. Clin Nephrol 2003; 60: S93-102. (Review)

56. Dries DL, Exner DV, Domanski MJ, Greenberg B, Stevenson LW. The prognostic implications of renal insufficiency in asymptomatic and symptomatic patients with left ventricular systolic dysfunction. J Am Coll Cardiol 2000; 35: 681-9.

57. Hillege HL, Girbes AR, de Kam PJ, et al. Renal function, neurohormonal activation, and survival in patients with chronic heart failure. Circulation 2000; 102: 203-10.

58. Laterza OF, Price CP, Scott MG. Cystatin C: an improved estimator of glomerular filtration rate? Clin Chem 2002; 48: 699-707. (Review)

59. Shlipak MG, Sarnak MJ, Katz R, et al. Cystatin C and the risk of death and cardiovascular events among elderly persons. N Engl J Med 2005; 352: 2049-60.

60. Sarnak MJ, Katz R, Stehman-Breen CO, et al. Cystatin C concentration as a risk factor for heart failure in older adults. Ann Intern Med 2005; 142: 497-505.

61. Arimoto T, Takeishi Y, Niizeki T, et al. Cystatin C, a novel measure of renal function, is an independent predictor of cardiac events in patients with heart failure. J Card Fail 2005; 11: 595-601.

62. Niizeki T, Takeishi Y, Arimoto T, et al. Combination of heart-type fatty acid binding protein and brain natriuretic peptide can reliably risk stratify patients hospitalized for chronic heart failure. Circ J 2005; 69: 922-7.

63. Ahmad T, Fiuzat M, Neely B, et al. Biomarkers of myocardial stress and fibrosis as predictors of mode of death in patients with chronic heart failure. JACC Heart Fail 2014; 2: 260-8.

64. Wiesner R, Edwards E, Freeman R, et al. Model for end-stage liver disease (MELD) and allocation of donor livers. Gastroenterology 2003; 124: 91-6.

65. Yang JA, Kato TS, Shulman BP, et al. Liver dysfunction as a predictor of outcomes in patients with advanced heart failure requiring ventricular assist device support: Use of the Model of Endstage Liver Disease (MELD) and MELD eXcluding INR (MELDXI) scoring system. J Heart Lung Transplant 2012; 31: 601-10.

66. Abe S, Yoshihisa A, Takiguchi M, et al. Liver dysfunction assessed by model for end-stage liver disease excluding INR (MELD-XI) scoring system predicts adverse prognosis in heart failure. PLoS One 2014; 9: e100618.

67. van Rooij E, Sutherland LB, Liu N, et al. A signature pattern of stress-responsive microRNAs that can evoke cardiac hypertrophy and heart failure. Proc Natl Acad Sci U S A 2006; 103: 18255-60.

68. Mitchell PS, Parkin RK, Kroh EM, et al. Circulating microRNAs as stable blood-based markers for cancer detection. Proc Natl Acad Sci U S A 2008; 105: 10513-8.

69. Chen X, Ba Y, Ma L, et al. Characterization of microRNAs in serum: a novel class of biomarkers for diagnosis of cancer and other diseases. Cell Res 2008; 18: 997-1006.

70. Tijsen AJ, Creemers EE, Moerland PD, et al. MiR423-5p as a circulating biomarker for heart failure. Circ Res 2010; 106: 1035-9.

71. Goren Y, Kushnir M, Zafrir B, Tabak S, Lewis BS, Amir O. Serum levels of microRNAs in patients with heart failure. Eur J Heart Fail 2012; 14: 147-54.

72. Tutarel O, Dangwal S, Bretthauer J, et al. Circulating miR-423 5p fails as a biomarker for systemic ventricular function in adults after atrial repair for transposition of the great arteries. Int $\mathbf{J}$ Cardiol 2013; 167: 63-6.

73. Corsten MF, Dennert R, Jochems S, et al. Circulating microRNA208b and microRNA-499 reflect myocardial damage in cardiovascular disease. Circ Cardiovasc Genet 2010; 3: 499-506.

74. Ellis KL, Cameron VA, Troughton RW, Frampton CM, Ellmers LJ, Richards AM. Circulating microRNAs as candidate markers to distinguish heart failure in breathless patients. Eur J Heart Fail 2013; 15: 1138-47.

75. Cheng JM, Akkerhuis KM, Battes LC, et al. Biomarkers of heart failure with normal ejection fraction: a systematic review. Eur J Heart Fail 2013; 15: 1350-62. (Review)

76. Zile MR, Desantis SM, Baicu CF, et al. Plasma biomarkers that reflect determinants of matrix composition identify the presence of left ventricular hypertrophy and diastolic heart failure. Circ Heart Fail 2011; 4: 246-56.

77. Yamaguchi H, Yoshida J, Yamamoto K, et al. Elevation of plasma brain natriuretic peptide is a hallmark of diastolic heart failure independent of ventricular hypertrophy. J Am Coll Cardiol 2004; 43: 55-60.

78. Kitahara T, Takeishi Y, Arimoto T, et al. Serum carboxy-terminal telopeptide of type I collagen (ICTP) predicts cardiac events in chronic heart failure patients with preserved left ventricular systolic function. Circ J 2007; 71: 929-35.

79. Krum H, Elsik M, Schneider HG, et al. Relation of peripheral collagen markers to death and hospitalization in patients with heart failure and preserved ejection fraction: results of the I-PRESERVE collagen substudy. Circ Heart Fail 2011; 4: 561-8.

80. Ahmad T, Fiuzat M, Pencina MJ, et al. Charting a Roadmap for Heart Failure Biomarker Studies. JACC Heart Fail (in press) 